\title{
Temporal flood incidence forecasting for Segamat River (Malaysia) using autoregressive integrated moving average modelling
}

\begin{abstract}
Accurate and efficient flood forecasting system can improve the emergency rescue plans and help avoid the loss of lives. This study aims to identify the trends in rainfall and streamflow in Segamat River (Malaysia) by using Mann-Kendall trend analysis, to develop time series flood forecasting model by the application of autoregressive integrated moving average (ARIMA) modelling approach. The accuracy of the optimal ARIMA model was verified by Spearman's rank correlation and linear regression analysis. The best $\operatorname{ARIMA~model~was~} \operatorname{ARIMA}(0,1,2)$. Trend analysis indicates that there was a trend of significant increase in rainfall rates at Kemelah Station and significant decrease at the Bandar Segamat Station, whereas streamflows at Bandar Segamat showed a trend of significant decrease. There was also a trend of decrease in streamflow over the study period. The applications of statistical modelling are beneficial to relevant authorities in understanding the flood patterns, trends and their potential risk.
\end{abstract}

Keyword: ARIMA model; Food; Mann-Kendall trend analysis; Rainfall; Segamat; Spearman's rank correlation analysis; Statistics; Streamflow 